\title{
A novel approach for part family formation using $\boldsymbol{K}$-means algorithm
}

\author{
Ashutosh Gupta $\cdot$ P. K. Jain • Dinesh Kumar
}

Received: 2 March 2013 / Accepted: 20 June 2013/Published online: 10 July 2013

(C) Shanghai University and Springer-Verlag Berlin Heidelberg 2013

\begin{abstract}
The reconfigurable manufacturing system (RMS) is the next step in manufacturing, allowing the production of any quantity of highly customized and complex parts together with the benefits of mass production. In RMSs, parts are grouped into families, each of which requires a specific system configuration. Initially system is configured to produce the first family of parts. Once it is finished, the system will be reconfigured in order to produce the second family, and so forth. The effectiveness of a RMS depends on the formation of the optimum set of part families addressing various reconfigurability issues. The aim of this work is to establish a methodology for grouping parts into families for effective working of RMS. The methodology carried out in two phases. In the first phase, the correlation matrix is used as similarity coefficient matrix. In the second phase, agglomerative hierarchical $K$-means algorithm is used for the parts family formation resulting in an optimum set of part families for reconfigurable manufacturing system.
\end{abstract}

Keywords Reconfigurable manufacturing system (RMS) . Part family formation $\cdot K$-means algorithm $\cdot$ Similarity coefficient $\cdot$ Correlation matrix

\section{Introduction}

Current market trends are characterized by globalization, new product requirements, rapidly changing demands, and a continuous improvement of the existing technology for manufacturing activities. Time reduction of introducing new products to the market with high quality and low cost is a necessity for

A. Gupta $(\bowtie) \cdot$ P. K. Jain · D. Kumar

Mechanical and Industrial Engineering Department, Indian Institute of Technology Roorkee, Roorkee 247667, India

e-mail: ashuaec72@gmail.com enterprise survival in this new period. The key factor in this highly competitive environment is the ability of the companies to launch new products to the market with high quality and low cost. To achieve this, the manufacturing system must have exact capacity and functionality to yield different batch sizes of different product types. Koren et al. [1] defined reconfigurable manufacturing system (RMS) as "a manufacturing system designed at the outset for rapid changes in structure as well as in hardware and software components in order to quickly adjust production capacity and functionality within a part family in response to sudden changes in market or in regulatory requirements." It is evident from the definition that formation of part families is a central issue in RMS. The key attribute of part families is that all the components within a family require similar production systems. Thus RMSs should have the exact capacity and functionality required to manufacture a part family, allowing cost effectiveness [2]. It is suggested that grouping parts into families in RMSs has a positive effect on the introduction of new products in market [3].

Initially, RMS is configured to produce the first selected family, and then it is reconfigured to effectively produce the following part family, and so forth. Thus, the RMS configuration changes over a number of part families to complete all the batches. Literatures present plenty of methods to obtain families and diverse formation criteria. These methods and criteria cannot be used directly in reconfigurable manufacturing because it has its own singularities that are to be taken into consideration, which differ from other manufacturing paradigms. Galan et al. [4] stated that formation of part families in RMS has to be based on some grouping criteria.

For customized products, modularity allows the assembly of simple and functionally independent parts. These parts are having the similar type of operations which are grouped together on the basis of the similarity to take the advantages of 
the group technology (GT). The group analysis uses arraybased, hierarchical and non hierarchical clustering methods. The array-based clustering methods achieve acceptable solutions with low computational cost. Although ordered matrices can be developed using the array-based clustering, disjoint part families or machine groups are not identified [5]. The hierarchical clustering agglomerative methods group together similar elements (products) in clusters are based on their attribute similarities. The coefficients that measure similarity between two parts are calculated from the incidence matrix. After that, a dendrogram shows the similarity degree to group parts. These methods are the most broadly implemented. They use similarity or dissimilarity coefficients among parts to obtain the groups. The most important similarity coefficient for part-family formation is the Jaccard's similarity coefficient [6], which is further modified to the weighted similarity coefficient [7], the commonality score [8], other measures of similarity [9]. Vakharia and Wemmerlöv [10] elaborated the comparative investigation of hierarchical clustering techniques and dissimilarity measures applied to the cell formation problem.

Most of the existing group formation methods suffer from one or more drawbacks. Their major common drawbacks are the inflexibility and the limited industrial application due to the non-availability of software programs supporting them. Hence, new part family formation approaches that overcome these limitations are clearly needed.

Usually, data mining techniques are found suitable for the class description, association, correlation analysis, classification, prediction and cluster analysis. Cluster analysis is one of the major data analysis methods widely used for many practical applications in emerging areas. Clustering is the unsupervised learning technique used to find the groups of objects such that the objects in a group will be similar to one another and different from the objects in other groups. A good clustering method will produce high quality clusters with high intra-cluster similarity. The quality of a clustering result depends on both the similarity measure used by the method and its implementation. There exists a number of clustering algorithms among which the $K$-means algorithm $[11,12]$ is commonly used to find clusters due to its simplicity of implementation and fast execution. It appears extensively in the machine learning [13] and in most data mining suite of tools. In Ref. [14], $K$-means algorithm was used for part family formation problem and obtained the best grouping of parts into families such that the parts within each family are as similar to each other as possible.

The $K$-means algorithm is a clustering technique, and it randomly selects $K$ data points as initial cluster centroids. A centroid is an artificial point in the space which represents an average location of the particular cluster. $K$ clusters are formed by assigning each data point to its nearest centroid. New virtual centroids are then calculated for each cluster.
These processes are iterated until a predefined number of iteration is reached or the clusters did not change anymore. The major problem with the $K$-means algorithm is that its initial starting points are generated randomly and do not guarantee the unique clustering results [15]. In addition, due to the non-hierarchical nature of the algorithm, a hierarchical relationship between the clusters is needed. This hierarchical relationship is effective to visualize and analyze the large data sets. The hierarchical technique is classified into agglomerative method and divisive method. The divisive method is the top down approach in which initially all the objects are included in a single cluster. Then, the single cluster is divided into sub-clusters until each object constitutes a cluster. An agglomerative method is the bottom up approach in which each object is assumed as a separate cluster and then they are clustered in succession until a single cluster which consists of the entire object set is formed. Thus, modified agglomerative hierarchical $K$-means clustering algorithm (AHKCA) is used for the part family formation. To do this effect, a method has been proposed using correlation as a similarity coefficient followed by agglomerative hierarchical $K$-means for identifying part family on the basis of operational similarity.

The outline of the paper is as follows: The proposed model is presented in the Sect. 2 followed by the methodology for grouping parts into families in the Sect. 3. Afterwards, one numerical example gives illustration of the proposed methodology in phased manner in Sect. 4. Section 5 discusses the result, and the conclusions are drawn in Sect. 6.

\section{Proposed model}

Different types and quantities of parts must be manufactured within a certain time horizon in a reconfigurable manufacturing system, using the reconfigurable machine tools (RMTs) and their available modules. A reconfigurable system is configured with the necessary RMT and modules to manufacture a family of similar products at the same time. Once a family is manufactured, the system will be reconfigured for manufacturing the following family effectively. In each change, the system incurs a reconfigurable cost, which depends on the current configuration and the following configuration. The proposed model possesses the features as described below:

(i) Manufacturer receives orders for $Q$ different part types for manufacturing in the next reconfiguration cycle. The order receipt is closed at a certain pre-defined time limit before the completion of running reconfiguration cycle to provide sufficient time for the reconfiguration exercise. Out of the $Q$ part types, only those $P(\leq Q)$ part types are accepted which satisfy two conditions as per company policy: (1) the total order $d_{i}$ of all the customers for a particular part type $P_{i}$ must exceed a pre-decided minimum quantity, $D_{\min }(i)$ 


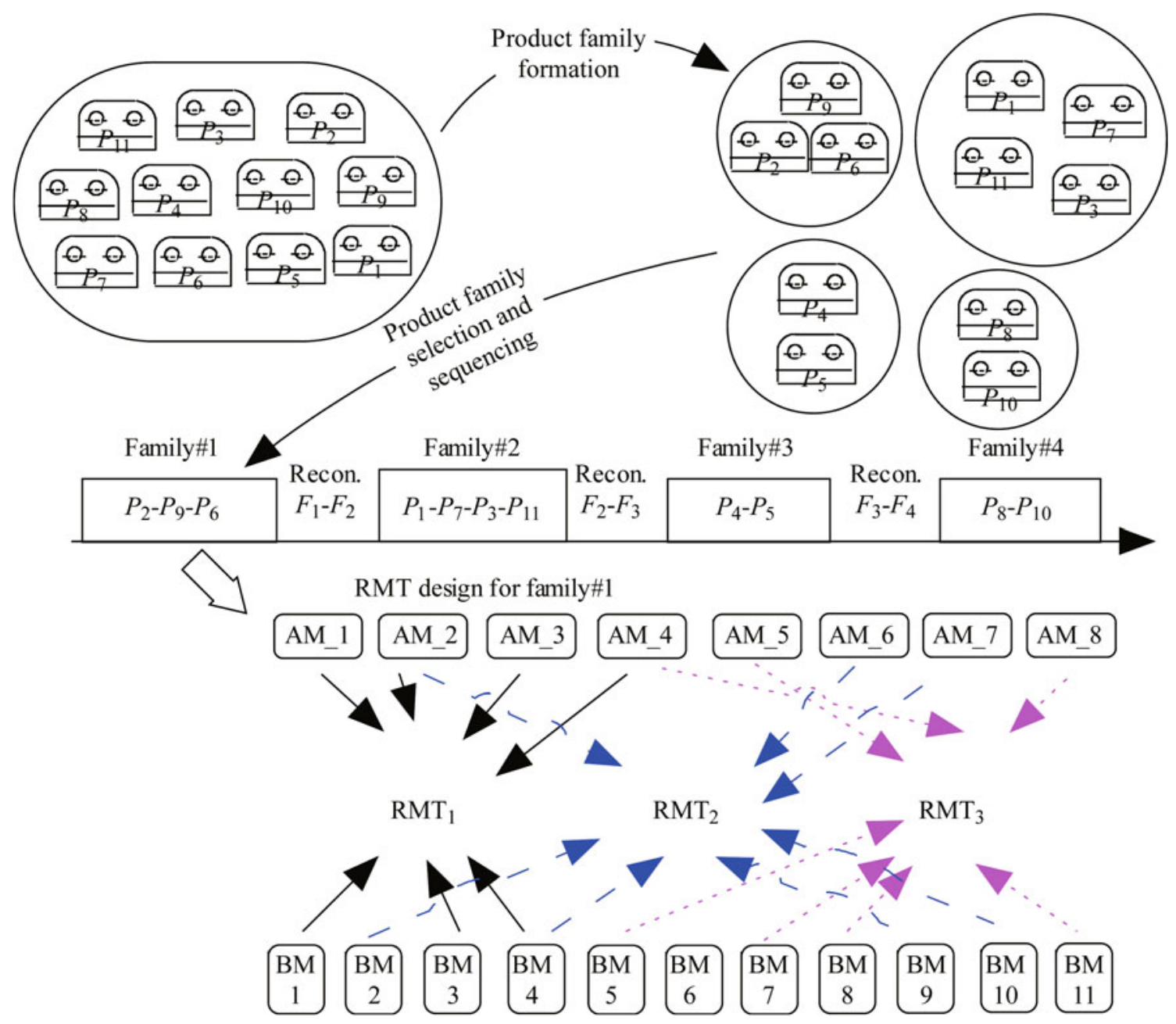

Fig. 1 The proposed approach

(i.e., $d_{i} \geq D_{\min }(i)$ ), and (2) the anticipated execution time of all the orders must not exceed a predefined maximum allowable reconfiguration cycle span.

(ii) The operations required to manufacture a part and their sequences are stated on a process plan. The plan so selected could be used for the manufacturing of full demand volume of the concerned part type. Therefore, it is being proposed that the system planner will have the selected process plan in hand for formation of part families.

(iii) Each part, $P_{i}$ is manufactured in ordered quantity, $d_{i}$ using various operations by one of the various alternative operation sequences. It has been proposed to give equal priority to all alternative operation sequences and to select only one operation sequence from the set of alternatives for the manufacturing of full demand volume of the concerned part.

(iv) These part types are divided into $K$ part families so that $1 \leq K \leq P$. Therefore, in extreme cases, either all the part types will fall in the same family or each family will be consisting of only one part type. The key attribute of a part family is that all the part types within a family require the same operations and hence the same production resources.

(v) A part is associated to a family based on the number of common operations that it shares with other members of the family. A reconfigurable machine tool has to remain idle when a member of the family does not require an operation. A higher value of similarity coefficient implies that a higher number of operations are common and therefore reduce the machine idleness. Therefore, it is proposed to choose operations based on the highest similarity coefficient. It implies that operation sequences similarity correlation will be beneficial to use in the clustering procedure and accordingly part family will be selected.

(vi) Selection and sequences of the product families with the minimum operational costs (that is, reconfiguration costs and the costs of under-utilization of resources).

Figure 1 shows a schematic representation of the proposed model approach, with $P_{i}$ (say $P_{1}, P_{2}, P_{3}, \cdots, P_{11}$ ) types of 
parts to be manufactured within a certain time horizon. Based on the proposed methodology (i.e., part families formation), $K$ families are formed (say $\left\{P_{2}-P_{6}-P_{9}\right\},\left\{P_{1}-P_{3}-\right.$ $\left.\left.P_{11}-P_{7}\right\},\left\{P_{4}-P_{5}\right\},\left\{P_{8}-P_{10}\right\}\right)$. Each part family require a different configuration of the RMS. These similar parts allow a reduction of setups, lead-times, work-in-process and material handling, thus increasing productivity. For each part family, the system is reconfigured, and the corresponding total cost are calculated to get the minimum cost solution for sequence of part families.

\section{Methodology}

In the proposed RMS model, it has been assumed that all the parts belonging to a family are manufactured on a system configuration that provides all the resources required for their manufacturing. All the parts are manufactured sequentially (one after the other) on the same system, and orders are accepted as per a predefined policy which ensures that the manufacturing time of all the parts does not exceed the cycle span. Therefore, demand data will not influence the decision of part family formation. It implies that for a part to be a member of a family, commonality of operations is a sufficient condition. Thus, the operations required to manufacture a part are the most relevant attributes for the considered RMS model, consistent with the objective of minimizing the number and hence, cost of reconfigurations. Therefore, binary part-operation incidence matrix (POIM) is the sufficient input for this problem. In this section, a methodology based on $K$-means algorithm is developed to form part families on the basis of the input received. The objective of this work is to cluster the parts into $K$ part families based on operations similarity. The proposed methodology consists of two stages, as shown in Fig. 2.

\subsection{Phase I: similarity coefficient matrix}

The first phase starts with building a similarity coefficient matrix. The initial part-operation incidence matrix as shown in Eq. (1) is a binary matrix in which rows represent the operations and columns stand for parts. This matrix looks like the transpose of the classical part-machine incidence matrix.

$\boldsymbol{A}=\left[\begin{array}{cccc}a_{11} & a_{12} & \cdots & a_{1 m} \\ \vdots & \vdots & \ddots & \vdots \\ a_{n 1} & a_{n 2} & \cdots & a_{n m}\end{array}\right]$

where the element of initial incidence matrix $a_{i j}=1$ if part $j$ requires operation $i$, otherwise $a_{i j}=0$. Hence the input parameters for the initial incidence matrix are parts and their operations sequence required for manufacturing.

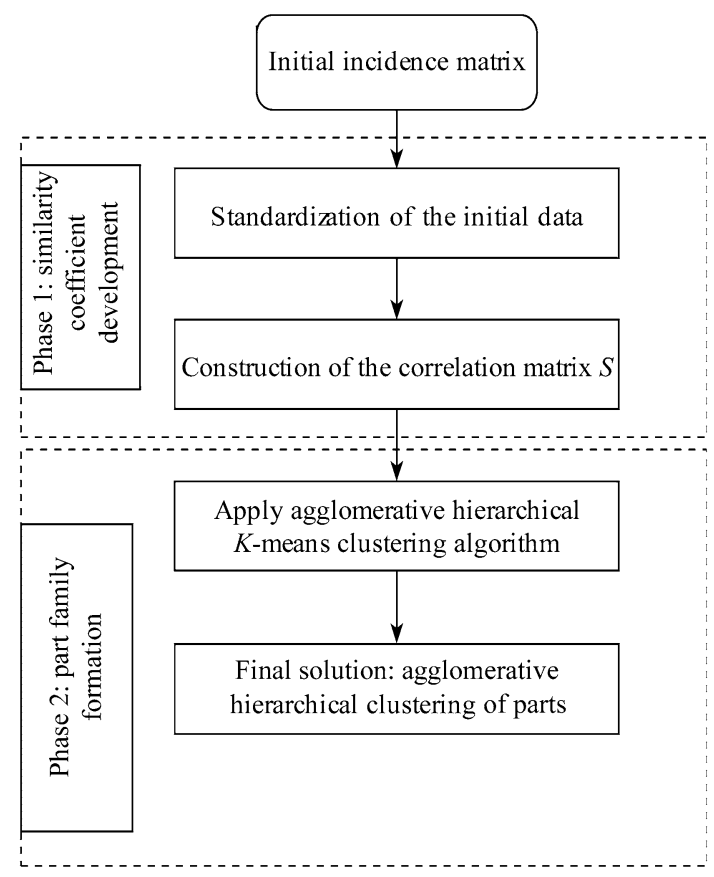

Fig. 2 Methodology

Let $\boldsymbol{P}_{j}^{A}$ is a binary row vector of matrix $\boldsymbol{A}$, such that $\boldsymbol{P}_{j}^{A}=\left[a_{1 j}, a_{2 j}, \cdots, a_{n j}\right]$. The initial matrix $\boldsymbol{A}$ is further standardized by using a suitable method of standardization. In this work, the general standardization is used for parts operations sequence and applied to the initial incidence matrix. The standardization process is expressed as follows:

$\boldsymbol{P}_{j}^{B}=\frac{\boldsymbol{P}_{j}^{A}-E_{j}}{\sigma_{j}}$,

where $E_{j}$ is the average of row vector $\boldsymbol{P}_{j}^{A}$, and $\boldsymbol{P}_{j}^{B}$ is the row vector of the standardized matrix $\boldsymbol{B} . E_{j}$ can be expressed as:

$E_{j}=\frac{\sum_{k=1}^{n} a_{k j}}{n}$,

where $a_{k j}$ is the element of initial incidence matrix, and $n$ is the number of elements in a row vector. Similarly,

$\sigma_{j}^{2}=\frac{1}{n} \sum_{k=1}^{n}\left(a_{k j}-E_{j}\right)^{2}$.

To simplify the Eq.(4), the Huyghens Koning theorem is applied to yield

$\sigma_{j}^{2}=\sqrt{E_{j}-\left(E_{j}\right)^{2}}$.

Once the standardized matrix $\boldsymbol{B}$ is formed, the proposed similarity coefficient will be based on the simple correlation matrix of the standard incidence matrix. The correlation matrix $\boldsymbol{S}$ is defined as follows: 
$\boldsymbol{S}=\frac{1}{n} \boldsymbol{B}^{\mathrm{T}} \boldsymbol{B}$

$S$ is the square matrix in which elements are given as

$S_{i i}=1$, and $S_{i j}=\frac{1}{n} \sum_{k=1}^{n} b_{i k} b_{j k}$.

\subsection{Phase II: algorithm for part family formation}

In the second phase of the proposed approach, the part family formation is done on the basis of operations similarity using correlation matrix defined in Eqs. (6) and (7). A modified AHKCA is used for this purpose resulted the part families formation. An agglomerative method is the bottom up approach in which each part is assumed as a separate cluster, and then they will be clustered in succession until a single cluster which consists of the entire parts is formed. This means that lateral shifting of object from one cluster to another cluster by violating the hierarchy is permitted. In this, initial seeds of the parts are taken based on certain value of the similarity coefficient. Each part should be present in any one of the initial seed. We implemented a modified form of the $K$-means algorithm, forming $K$ clusters at each level of a hierarchical tree. The flow chart of the modified algorithm is presented in the Fig. 3. The algorithm for assigning the $n$ parts (say $P_{i}$, where $i$ is from 1 to $n$ ) into parts families is given below. It may be noted that in the beginning, there is no cluster of the parts existence. Hence, it is assumed that each part represents a cluster with itself.

\section{Numerical illustration}

An example case has been taken to demonstrate the proposed methodology where seven operations are required for manufacturing of parts in next reconfiguration cycle. Table 1 shows the operation sequences, the total demand, $d_{i}$ and the minimum acceptable demand size $D_{\min }(i)$ for each part type. The required RMT are configured by two base module and maximum of three auxiliary module for the example problem. The machine $M_{1}$ is modular in structure for its first configuration $\left(M_{11}\right)$, requires two base module namely $\{01,02\}$ and three auxiliary module $\{01,03,05\}$ from the available set of base module $\{01,02,03,04,05, \cdots\}$ and set of auxiliary module $\{01,02,03,04,05,06,07,08,09,10,11, \cdots\}$ respectively. The machine $M_{2}$ for its configuration $\left(M_{21}\right)$ requires base module of $\{03,05\}$ and auxiliary module $\{08,10,11\}$. The machine is reconfigured to another configuration $\left(M_{23}\right)$ by keeping the base module same i.e., $\{03,05\}$, but change in the auxiliary modules from $\{08,10,11\}$ to $\{06,07,10\}$.Similarly the other machines can be reconfigured into others configurations depending upon the requirements of manufacturing. Table 2 shows the RMTs resources available for the manufacturing the parts.

From the above available data, POIM is constructed as shown in Table 3. Any order (which is less than minimum demand) $d_{i}<D_{\min }(i)$ is not considered, Part $P_{12}$ demand $d_{i}$ is 80 units which is less than the minimum acceptable demand $D_{\min }(i)$. Hence $P_{12}$ is not considered here. Thus, the POIM (see Table 3) has total 11 parts and 7 operations.

(i) For each part $P_{i}$, where $i=1$ to $n$,

Compute the distance of each part to all the centroids $C_{j}(1 \leq j \leq n)$ using Euclidean distance formula

(ii) For each part $P_{i}$, find the closest centroid $C_{j}$ and club $P_{i}$ to the cluster with the nearest centriod

$C_{j}$ and store them in array cluster [ ] and Dist[ ] separately.

Set cluster $[i]=j, j$ is the label of the nearest cluster

Set Dist $[i]=d\left(x_{i}, C_{j}\right), d\left(x_{i}, C_{j}\right)$ is the nearest Euclidean distance to the closest cluster.

(iii) For each cluster $j(1 \leq j \leq n)$, recalculate the centroids

(iv) Repeat

(v) For each part

(1) Compute its distance from the centroid of the present nearest cluster

(2) If this distance is less than or equal to the previous nearest distance, the part stay in the cluster else

For every centroid $C_{j}$

Compute the distance of each part to all the centre club the part to the cluster with the nearest centroid $C_{j}$

(vi) For each cluster $C_{j}(1 \leq j \leq n)$, recalculate the distance, until all the parts are clubbed

(vii) Draw the dendrogram of parts 


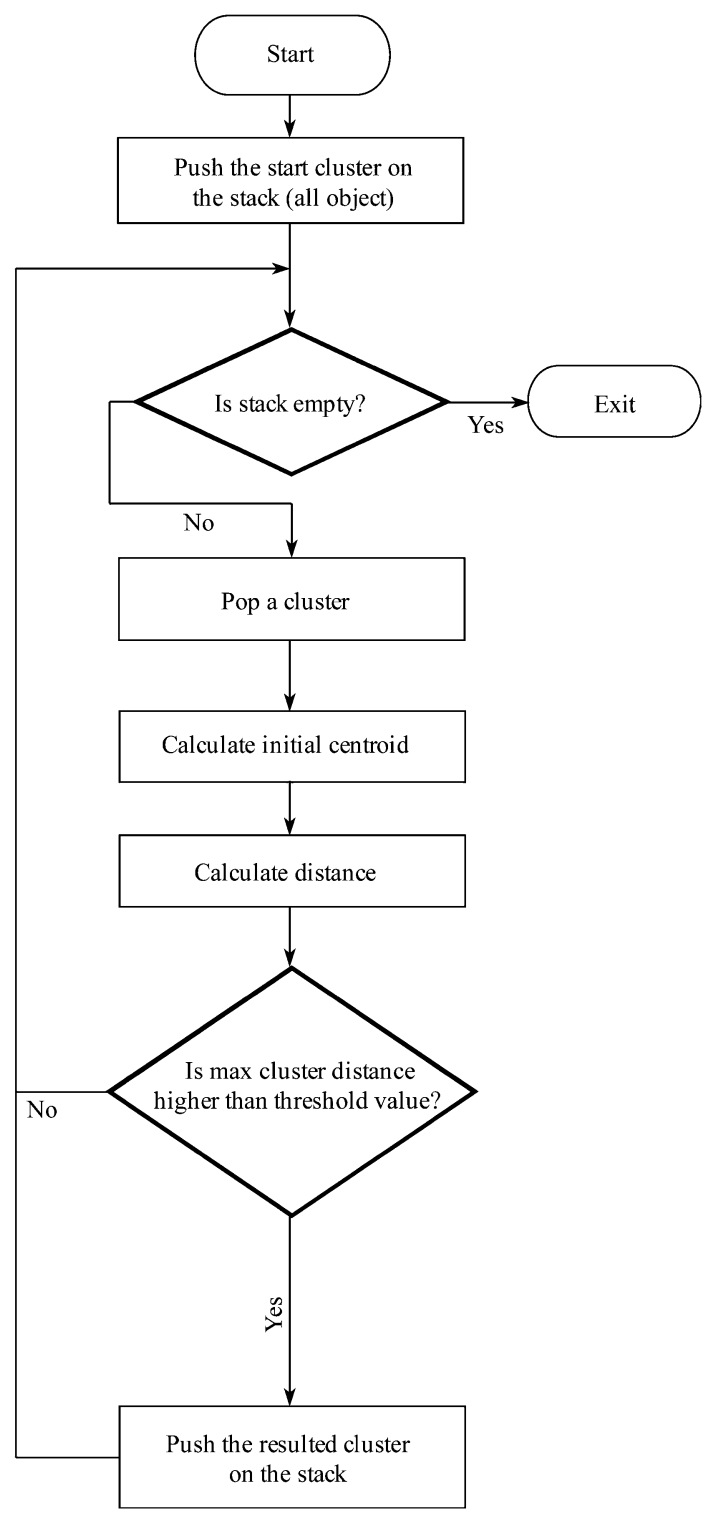

Fig. 3 Algorithm flow chart for parts family formation

Then applying Eqs. (2), (3) and (5) to the part operation incidence matrix given above, yield standardized matrix $\boldsymbol{B}$, which represents standard sequence part operation matrix (SSPOM) in the case. The following procedure is adopted to determine various elements of SSPOM.

For the part $P_{1}$,

$E_{1}=2 / 7=0.286$,

$\sigma_{1}=\sqrt{0.286-(0.286)^{2}}=0.452$.

The member coefficient between $P_{1}$ and $\mathrm{OP}_{1}$ (i.e., $\mathrm{b}_{11}$ ) is calculated as follows:

$b_{11}=\frac{1-0.286}{0.452}=1.580$.
Table 1 Parts demand data and operations sequence with operation time

\begin{tabular}{lrrl}
\hline $\begin{array}{l}\text { Part } \\
\text { No. }\end{array}$ & $D_{\min }(i)$ & Demand $\left(d_{i}\right)$ & $\begin{array}{l}\text { Operation sequence with operation } \\
\text { time }\end{array}$ \\
\hline$P_{1}$ & 40 & 60 & $\mathrm{OP}_{1}(140)-\mathrm{OP}_{2}(115)$ \\
$P_{2}$ & 100 & 150 & $\mathrm{OP}_{2}(150)-\mathrm{OP}_{3}(135)$ \\
$P_{3}$ & 100 & 250 & $\mathrm{OP}_{1}(85)-\mathrm{OP}_{5}(100)-\mathrm{OP}_{6}(65)$ \\
$P_{4}$ & 50 & 80 & $\mathrm{OP}_{4}(70)-\mathrm{OP}_{5}(65)-\mathrm{OP}_{6}(55)-\mathrm{OP}_{7}(60)$ \\
$P_{5}$ & 50 & 100 & $\mathrm{OP}_{4}(75)-\mathrm{OP}_{5}(85)-\mathrm{OP}_{6}(95)$ \\
$P_{6}$ & 100 & 150 & $\mathrm{OP}_{2}(150)-\mathrm{OP}_{3}(135)$ \\
$P_{7}$ & 100 & 120 & $\mathrm{OP}_{1}(90)-\mathrm{OP}_{6}(110)$ \\
$P_{8}$ & 120 & 180 & $\mathrm{OP}_{6}(145)-\mathrm{OP}_{7}(105)$ \\
$P_{9}$ & 70 & 110 & $\mathrm{OP}_{3}(160)$ \\
$P_{10}$ & 90 & 160 & $\mathrm{OP}_{5}(95)-\mathrm{OP}_{6}(75)-\mathrm{OP}_{7}(80)$ \\
$P_{11}$ & 100 & 200 & $\mathrm{OP}_{1}(125)-\mathrm{OP}_{5}(130)$ \\
$P_{12}$ & 120 & 80 & $\mathrm{OP}_{6}(100)-\mathrm{OP}_{7}(130)$ \\
\hline
\end{tabular}

Table 2 RMT resources required for manufacturing

\begin{tabular}{llll}
\hline Machine & $\begin{array}{l}\text { Machine } \\
\text { module }\end{array}$ & $\begin{array}{l}\text { Basic module } \\
(\mathrm{BM})\end{array}$ & $\begin{array}{l}\text { Auxiliary module } \\
(\mathrm{AM})\end{array}$ \\
\hline$M_{1}$ & $M_{11}$ & $\{01,02\}$ & $\{01,03,05\}$ \\
$M_{2}$ & $M_{21}$ & $\{03,05\}$ & $\{08,10,11\}$ \\
& $M_{22}$ & $\{03,05\}$ & $\{08,05,07\}$ \\
& $M_{23}$ & $\{03,05\}$ & $\{06,07,10\}$ \\
$M_{3}$ & $M_{31}$ & $\{01,04\}$ & $\{02,09\}$ \\
& $M_{32}$ & $\{01,04\}$ & $\{02,09,11\}$ \\
& $M_{33}$ & $\{01,04\}$ & $\{02,09,10\}$ \\
$M_{4}$ & $M_{41}$ & $\{02,05\}$ & $\{03,08\}$, \\
$M_{5}$ & $M_{51}$ & $\{03,06\}$ & $\{04,09,10\}$ \\
& $M_{52}$ & $\{03,06\}$ & $\{04,07,09\}$, \\
$M_{6}$ & $M_{61}$ & $\{04,07\}$ & $\{01,03,07\}$ \\
& $M_{62}$ & $\{04,07\}$ & $\{01,03\}$ \\
\hline
\end{tabular}

Table 3 Part operation incidence matrix: POIM A

\begin{tabular}{llllllllllll}
\hline & $P_{1}$ & $P_{2}$ & $P_{3}$ & $P_{4}$ & $P_{5}$ & $P_{6}$ & $P_{7}$ & $P_{8}$ & $P_{9}$ & $P_{10}$ & $P_{11}$ \\
\hline $\mathrm{OP}_{1}$ & 1 & 0 & 1 & 0 & 0 & 0 & 1 & 0 & 0 & 0 & 1 \\
$\mathrm{OP}_{2}$ & 1 & 1 & 0 & 0 & 0 & 1 & 0 & 0 & 0 & 0 & 0 \\
$\mathrm{OP}_{3}$ & 0 & 1 & 0 & 0 & 0 & 1 & 0 & 0 & 1 & 0 & 0 \\
$\mathrm{OP}_{4}$ & 0 & 0 & 0 & 1 & 1 & 0 & 0 & 0 & 0 & 0 & 0 \\
$\mathrm{OP}_{5}$ & 0 & 0 & 1 & 1 & 1 & 0 & 0 & 0 & 0 & 1 & 1 \\
$\mathrm{OP}_{6}$ & 0 & 0 & 1 & 1 & 1 & 0 & 1 & 1 & 0 & 1 & 0 \\
$\mathrm{OP}_{7}$ & 0 & 0 & 0 & 1 & 0 & 0 & 0 & 1 & 0 & 1 & 0 \\
\hline
\end{tabular}

Similarly,

$b_{21}=\frac{1-0.286}{0.452}=1.580$, and $b_{31}=\frac{0-0.286}{0.452}=-0.633$. 
The same procedure is repeated for other elements. Finally, SSPOM is obtained as shown in Table 4.

From the above SSPOM and on the basis of proposed similarity coefficient (as given in the Eq. (7)), the correlation matrix $S$ is obtained as shown in Table 5. The following procedure is adopted to determine the elements of the correlation matrix $S$. For similarity between $P_{1}$ and $P_{2}$

$S_{11}=1$ in the methodology. The algorithm is initialized by taking correlation values of parts from correlation matrix. The algorithm starts with the initial solution i.e., the number of parts and then computes and stores the Euclidean distance between each part. The parts having minimum distance are the grouped first, and these grouped parts are removed from the subsequent iteration. This process continues until all the parts are grouped together. The algorithm finally provides the output in the form of dendrogram as presented in Fig. 4.

$$
\begin{aligned}
& S_{12}=\frac{1}{7\left(\begin{array}{c}
(1.580 \times(-0.633))+(1.580 \times 1.580)+(-0.633 \times 1.580)+(-0.633 \times(-0.633)) \\
+(-0.633 \times(-0.633))+(-0.633 \times(-0.633))+(-0.633 \times(-0.633))
\end{array}\right)}=0.300, \\
& S_{13}=\frac{1}{7\left(\begin{array}{c}
(1.580 \times 1.154)+(1.580 \times(-0.867))+(-0.633 \times(-0.867))+(-0.633 \times(-0.867)) \\
+(-0.633 \times 1.154)+(-0.633 \times 1.154)+(-0.633 \times(-0.867))
\end{array}\right)}=0.091 .
\end{aligned}
$$

The same procedure is repeated for the other elements for correlation matrix $\boldsymbol{S}$. The elements of the correlation matrix $S$ give the correlation between the various parts. In the example problem parts $P_{2}$ and $P_{6}$ are produced by similar type of operations sequence and required similar resources. Hence, they are perfectly correlated and magnitude of correlation is $0.999(\approx 1)$ (see Table 5). On the contrary parts $P_{2}$ and $P_{3}$ require dissimilar operations sequences, hence they are negatively correlated to each other. Similarly each part is either positively or negatively correlated with other parts depending upon the similarity in their operations sequence. The magnitude of similarity coefficient decides the correlation between the parts. This resulted correlation matrix is used in the second phase to get the optimal solution of the problem.

The second phase of the methodology is to cluster parts, obtained from the solution space in the first phase i.e., correlation matrix, into family by using AHKCA as mentioned

\section{Result and discussions}

The resulting dendrogram is based on the distances to $K$ means nearest group. In $K$-means algorithm, the measured distance is known as dissimilarity measure. Hence, the magnitude of the distance to nearest group of parts represents the dissimilarity associated with the part. The ratio of the distance to the nearest group to the maximum distance measured in the dendrogram gives the percentage of dissimilarity of the particular parts group. In Fig. 4, parts $\mathrm{P}_{2}$ and part $\mathrm{P}_{6}$ are found at zero distance. So their percentage of dissimilarity is zero. It means that they are $100 \%$ similar in operations. The distance between parts $\mathrm{P}_{4}$ and $\mathrm{P}_{5}$ is 0.60 . Their associated percentage of dissimilarity is calculated to $24.0 \%$ and the level of similarity is $76 \%$. The same procedure is repeated to calculate the other similarity level for remaining cluster of parts. The obtained results are summarized in Table 6.

Table 4 Standard sequence part operation matrix: SSPOM B

\begin{tabular}{lrrrrrrrrrrr}
\hline & \multicolumn{1}{l}{$P_{1}$} & \multicolumn{1}{c}{$P_{2}$} & \multicolumn{1}{c}{$P_{3}$} & \multicolumn{1}{c}{$P_{4}$} & \multicolumn{1}{c}{$P_{5}$} & \multicolumn{1}{c}{$P_{6}$} & \multicolumn{1}{c}{$P_{7}$} & $P_{8}$ & $P_{9}$ & $P_{10}$ & $P_{11}$ \\
\hline $\mathrm{OP}_{1}$ & 1.580 & -0.633 & 1.154 & -0.633 & -0.633 & -0.633 & 1.580 & -0.409 & -0.409 & -0.633 & 1.580 \\
$\mathrm{OP}_{2}$ & 1.580 & 1.580 & -0.867 & -0.633 & -0.633 & 1.580 & -0.633 & -0.409 & -0.409 & -0.633 & -0.633 \\
$\mathrm{OP}_{3}$ & -0.633 & 1.580 & -0.867 & -0.633 & -0.633 & 1.580 & -0.633 & -0.409 & 2.449 & -0.633 & -0.633 \\
$\mathrm{OP}_{4}$ & -0.633 & -0.633 & -0.867 & 1.580 & 1.580 & -0.633 & -0.633 & -0.409 & -0.409 & 1.580 & -0.633 \\
$\mathrm{OP}_{5}$ & -0.633 & -0.633 & 1.154 & -0.633 & -0.633 & -0.633 & 1.580 & -0.409 & -0.409 & -0.633 & -0.633 \\
$\mathrm{OP}_{6}$ & -0.633 & -0.633 & 1.154 & 1.580 & 1.580 & -0.633 & -0.633 & -0.409 & -0.409 & -0.633 & 1.580 \\
$\mathrm{OP}_{7}$ & -0.633 & -0.633 & -0.867 & -0.633 & -0.633 & -0.633 & -0.633 & 2.449 & -0.409 & 1.580 & -0.633 \\
\hline
\end{tabular}


Table 5 Correlation matrix: $S$

\begin{tabular}{|c|c|c|c|c|c|c|c|c|c|c|c|}
\hline & $P_{1}$ & $P_{2}$ & $P_{3}$ & $P_{4}$ & $P_{5}$ & $P_{6}$ & $P_{7}$ & $P_{8}$ & $P_{9}$ & $P_{10}$ & $P_{11}$ \\
\hline$P_{1}$ & 1.000 & & & & & & & & & & \\
\hline$P_{2}$ & 0.300 & 1.000 & & & & & & & & & \\
\hline$P_{3}$ & 0.091 & -0.548 & 1.000 & & & & & & & & \\
\hline$P_{4}$ & -0.200 & -0.400 & 0.091 & 1.000 & & & & & & & \\
\hline$P_{5}$ & 0.400 & -0.400 & 0.091 & 0.949 & 1.000 & & & & & & \\
\hline$P_{6}$ & 0.300 & 0.999 & -0.548 & -0.400 & -0.400 & 1.000 & & & & & \\
\hline$P_{7}$ & 0.300 & -0.400 & 0.730 & -0.400 & -0.400 & -0.400 & 1.000 & & & & \\
\hline$P_{8}$ & -0.258 & -0.258 & -0.354 & -0.258 & -0.258 & -0.258 & -0.258 & 1.000 & & & \\
\hline$P_{9}$ & -0.258 & 0.645 & -0.354 & -0.258 & -0.258 & 0.645 & -0.258 & -0.167 & 1.000 & & \\
\hline$P_{10}$ & -0.200 & -0.400 & -0.548 & 0.300 & 0.300 & -0.400 & -0.400 & 0.645 & -0.258 & 1.000 & \\
\hline$P_{11}$ & 0.300 & -0.400 & 0.730 & 0.300 & 0.300 & -0.400 & 0.300 & -0.258 & -0.258 & -0.400 & 1.000 \\
\hline
\end{tabular}

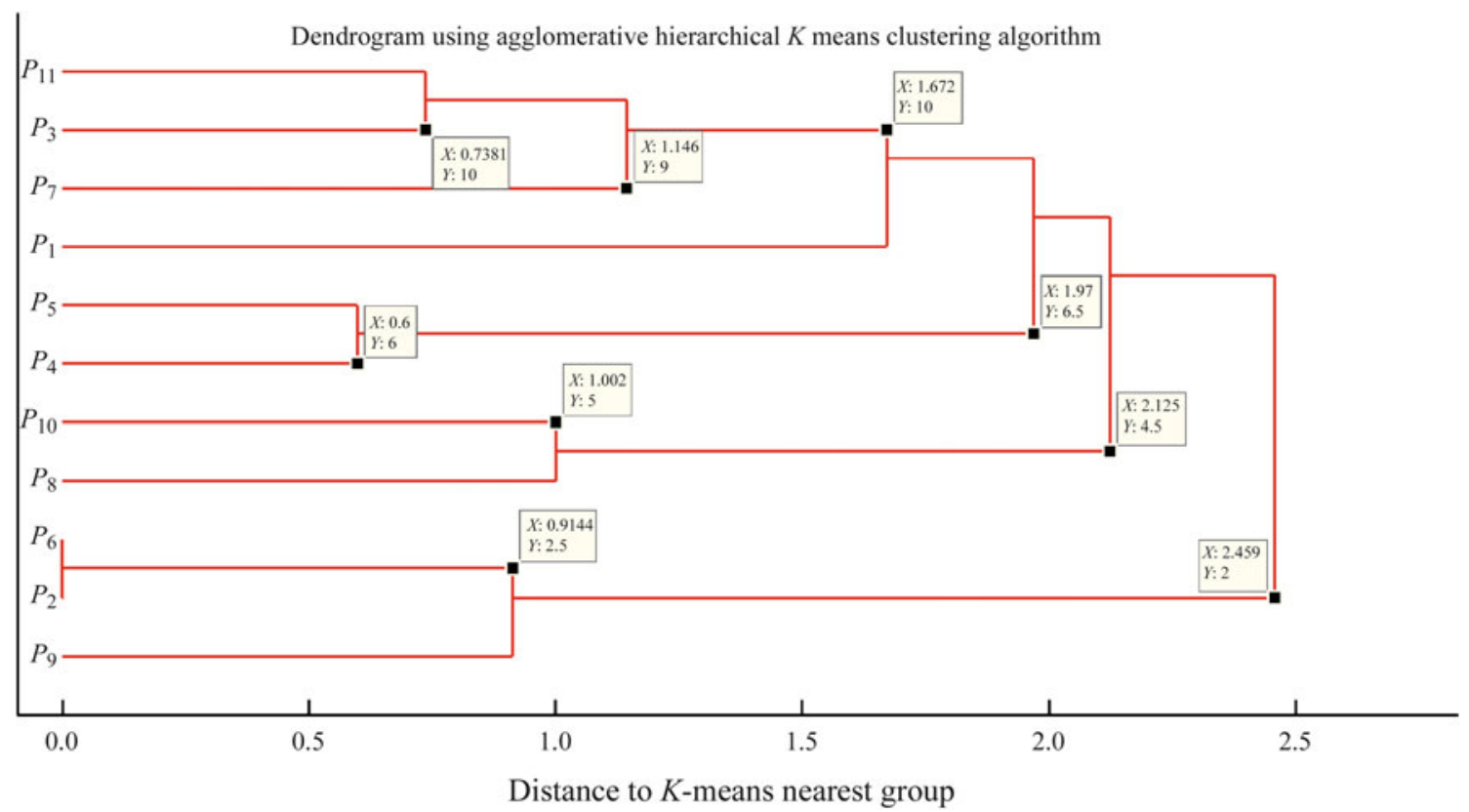

Fig. 4 Hierarchical clustering of parts

The dendrogram shown in Fig. 4 presents the different families that are formed, and their associated similarity level is presented in Table 6. At level 1, 10 families are formed named $P_{2,6}, P_{1}, P_{3}, P_{4}, P_{5}, P_{7}, P_{8}, P_{9}, P_{10}, P_{11}$, where part $P_{2}$, and $P_{6}$, are formed one family due to $100 \%$ similar in operations. At level 2, a set of 9 families are formed, out of which two families composed of parts $P_{2}$, $P_{6} ; P_{4}, P_{5}$ at $76 \%$ similarity and rest are composed of one part (i.e., $\left.P_{1}, P_{3}, P_{7}, P_{8}, P_{9}, P_{10}, P_{11}\right)$. Similarly for different level, others set of part families are obtained. Finally, at level $(L) 10$ only one family is formed composed of all parts at the lowest similarity in operations.

The adopted methodology provided the formation of parts family on the basis of operational similarity. There are methods available in the literature for formation of parts into families. They used different similarity coefficients to obtain the parts group, among which Jaccard's similarity coefficient is commonly used and obtained the optimum grouping of parts into families on the basis of operations similarity. Thus, for validating the proposed methodology, it is appropriate to solve the same example problem again using the Jaccard's similarity coefficient and compare the results. The proposed approach and the approach used for the validation provided the same set of part families at 100 similarity level $\left(L_{1}\right)$. But for similarity level (i.e. from $L_{2}$ to $L_{9}$ ), the proposed approach provided the better results for the set of part families at that particular level. The comparison between two approaches in 
Table 6 Percentage of similarity and formed part family

\begin{tabular}{|c|c|c|c|c|}
\hline Level & Distance between parts group & Similarity $/ \%$ & Formed part families & Number of families \\
\hline$L_{1}$ & 0.0000 & 100 & $\begin{array}{l}P_{2,6}, P_{1}, P_{3}, P_{4}, P_{5}, \\
P_{7}, P_{8}, P_{9}, P_{10}, P_{11}\end{array}$ & 10 \\
\hline$L_{2}$ & 0.6000 & 76.0 & $\begin{array}{l}P_{2,6}, P_{1}, P_{3}, P_{4,5}, P_{7}, P_{8} \\
P_{9}, P_{10}, P_{11}\end{array}$ & 09 \\
\hline$L_{3}$ & 0.7381 & 70.5 & $\begin{array}{l}P_{2,6}, P_{1}, P_{3,11}, P_{4,5} \\
P_{7}, P_{8}, P_{9}, P_{10}\end{array}$ & 08 \\
\hline$L_{4}$ & 0.9144 & 63.4 & $P_{2,6,9}, P_{1}, P_{3,11}, P_{4,5}, P_{7}, P_{8}, P_{10}$ & 07 \\
\hline$L_{5}$ & 1.0020 & 59.9 & $P_{2,6,9}, P_{1}, P_{3,11}, P_{4,5}, P_{7}, P_{8,10}$ & 06 \\
\hline$L_{6}$ & 1.1460 & 54.1 & $P_{2,6,9}, P_{1}, P_{3,11,7}, P_{4,5}, P_{8,10}$ & 05 \\
\hline$L_{7}$ & 1.6720 & 33.1 & $P_{2,6,9}, P_{1,3,11,7}, P_{4,5}, P_{8,10}$ & 04 \\
\hline$L_{8}$ & 1.9700 & 21.2 & $P_{2,6,9}, P_{1,3,11,7,4,5}, P_{8,10}$ & 03 \\
\hline$L_{9}$ & 2.1250 & 15.0 & $P_{2,6,9}, P_{1,3,11,7,4,5,8,10}$ & 02 \\
\hline$L_{10}$ & 2.4590 & 1.0 & $P_{2,6,9,1,3,11,7,4,5,8,10}$ & 01 \\
\hline
\end{tabular}

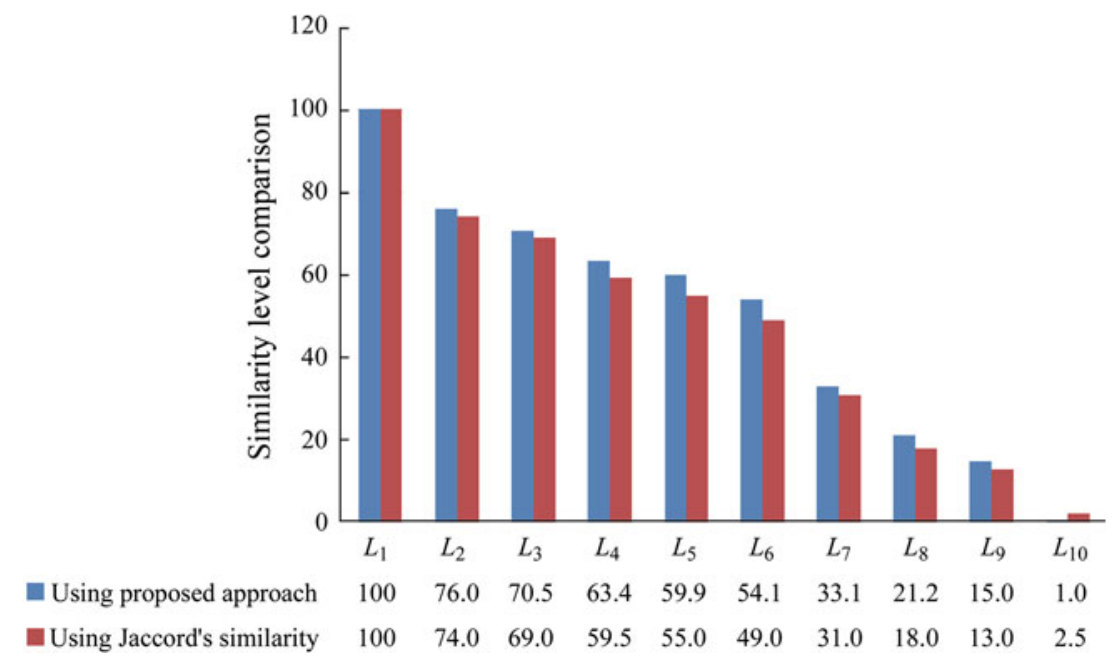

Fig. 5 Comparison of similarity level

terms of similarity level is presented in the Fig. 5. It shows that the proposed approach gives the better solution for part family formation. As the proposed approach used the modified $K$ means algorithm which is usually adopted for the data mining for large size data. Hence this proposed approach can easily handle the large size real life problem which is very difficult to handle by the any of the conventional approaches and can encourage the researchers and practitioner industrial engineers to adopt the proposed methodology.

On the basis of the formed part families, the system planner first configures the manufacturing system to produce the first part family. Each system reconfiguration adds cost to the production of the parts. Hence there is a need to arrive at a suitable number of part families by selecting the dendogram level and the sequencing of the corresponding families to achieve the minimum cost solution of the problem.

\section{Conclusions}

This work has presented a novel methodology for grouping parts into families on the basis of operation similarity which is a central issue in the design of reconfigurable manufacturing systems. A correlation analysis model is formulated to group the parts on the basis of operations similarity and correlation matrix is used as the similarity coefficient matrix. Finally, agglomerative hierarchical $K$ means algorithm is applied to find the level of similarity in parts. The obtained part families are based on compactness of the family formation on the basis of operational similarity. Hence, one can be easily applied the methodology to the other manufacturing system like cellular manufacturing system, etc. However the proposed work can be further extended to accommodate other factors such as, production volume, operation sequence and alternative routings. 


\section{References}

1. Koren Y, Jovane F, Heisel U et al (1999) Reconfigurable manufacturing systems. Ann CIRP 48(2):527-540

2. Lokesh K, Jain PK (2011) A model and optimization approach for reconfigurable manufacturing system configuration design. Int J Prod Res 50(12):3359-3381

3. Abdi MR, Labib AW (2004) Grouping and selecting products: the design key of reconfigurable manufacturing systems (RMSs). Int J Prod Res 42(3):521-546

4. Galan R, Racero J, Eguia I et al (2007) A systematic approach for product families formation in reconfigurable manufacturing systems. Robot Comp Integr Manuf 23(5):489-502

5. Mahesh O, Srinivasan G (2002) Incremental cell formation considering alternative machines. Int J Prod Res 40(14):3291-3310

6. McAuley J (1972) Machine grouping for efficient production. Prod Eng 51(2):53-57

7. Mosier CT, Taube L (1985) Weighted similarity measure heuristics for the group technology machine clustering problem. Int $\mathbf{J}$ Manag Sci 13:577-583
8. Wei JC, Kern GM (1989) Commonality analysis: a linear clustering algorithm for group technology. Int $\mathrm{J}$ Prod Res 12: 2053-2062

9. Sarker BR, Islam KM (1999) Relative performances of similarity and dissimilarity measures. Comp Ind Eng 37:769-807

10. Vakharia AJ, Wemmerlöv U (1995) A comparative investigation of hierarchical clustering techniques and dissimilarity measures applied to the cell formation problem. J Oper Manag 13:117-138

11. Zalik KR (2008) An efficient $K$-means clustering algorithm. Pattern Recognit Lett 29:1385-1391

12. Jain AK (2010) Data clustering: 50 years beyond $K$-means. Pattern Recognit Lett 31:651-666

13. Tsai CF, Hsu YF, Lin CY et al (2009) Intrusion detection by machine learning: a review. Expert Syst Appl 36:11994-12000

14. Al-Sultan KS (1997) A hard clustering approach to the part family formation problem. Prod Plann Control 8(3):231-236

15. Shehroz SK, Ahmad A (2004) Cluster centre initialization algorithm for $K$-means clustering. Pattern Recogni Letter 25:1293-1302 